\title{
A Forest Disease and Insect Pests Model which has a Forest Age Structure with Disease Rate Change Functions Qiao-qi XU and Ding-jiang WANG ${ }^{a}{ }^{*}$
}

\author{
Department of Applied Mathematics,Zhejiang University of \\ Technology,Hangzhou,Zhejiang,310023,China \\ awangdingj@126.com \\ ${ }^{*}$ Corresponding author
}

\begin{abstract}
Keywords: Forest age structure, Disease rate change function, Threshold, Infected
\end{abstract} steady state, Stability.

\begin{abstract}
This communication mainly covers investigations on the dynamic characteristics of forest diseases and insect pests' model which has a forest age structure with disease rate change functions. Firstly, the age structure model with partial differential equation with the function of the course is established. Secondly, it proves the existence of non-infected and infected steady state model. Finally, the stability and asymptotic stability of the steady state are discussed, and the condition of local asymptotic stability is obtained.
\end{abstract}

\section{Introduction}

Bio-mathematics, as an interdisciplinary subject, is attracting a large number of researchers at home and abroad to this field and has achieved a lot of results. In recent years, the study of forest diseases and insect pests is one of the hot issues; many scholars are of common concern. However, most mathematical models for forest diseases and insect pests are described by ordinary differential equations. There is little literature on the dynamics model of infection age structure of forest, and there is little discussion of the disease rate change function. Therefore, this paper presents a dynamic model of forest diseases and insect pests with infection age structure of forest. On this basis, the existence of the non-infected and infected steady state of the model with disease rate change function and the condition of local asymptotic stability are discussed.

\section{A Model of Forest Pests and Diseases with Infection-age Structure}

Our model considers a population of uninfected trees, $T(t)$, the density distribution function of the trees by the age $a$ of their infection, $T^{*}(a, t)$, and pests, $V(t)$.at time $t$. In this model, the rate of planting trees, $A(A>0)$, and the rate of fallen trees, $\mu \quad(\mu>0)$,and the Infection coefficient of the trees that are infected with pests, $k \quad(k>0)$. Then we consider the rate of fallen trees by the age $a$ of their infection, $\delta(a) \quad\left(\delta(a) \in[0, N]\right.$. The boundary value function of $T^{*}(a, t)$ determined wherein, $T^{*}(0, t)=k V(t) T(t)$, it means the number of trees that have just been infected by the pest. Finally, we consider the pest breeding rate, $p(a)(p(a) \in[0, N])$, so that we can obtain the total number of pests on all infected trees by the age $a$ of their infection, $\int_{0}^{\infty} p(a) T^{*}(a, t) d a$, and the rate of removal of pests, $c \quad(c>0)$. We 
called $\varphi(a) \in[0, N]$ as "disease rate change function". Accordingly, we put forward the following forest disease and insect pest model with age function:

$$
\left\{\begin{array}{l}
\frac{d T(t)}{d t}=A-\mu T(t)-k V(t) T(t), \\
\frac{\partial \varphi(a) T^{*}(a, t)}{\partial a}+\frac{\partial T^{*}(a, t)}{\partial t}=-\delta(a) T^{*}(a, t), \\
T^{*}(0, t)=k V(t) T(t), \\
\frac{d V(t)}{d t}=\int_{0}^{\infty} p(a) T^{*}(a, t) d a-c V(t) .
\end{array}\right.
$$

\section{Stability Analysis}

To determine the steady states of the age-structured model, we solve

$$
\left\{\begin{array}{l}
A-\mu T-k V T=0, \\
\frac{d \varphi(a) T^{*}(a)}{d a}=-\delta(a) T^{*}(a), \\
T^{*}(0)=k V T, \\
\int_{0}^{\infty} p(a) T^{*}(a) d a=c V .
\end{array}\right.
$$

It is easily seen that one steady state is the non-infected steady state

$$
\left(T_{s s}, T^{*}(a)_{s s}, V_{s s}\right)=\left(\frac{A}{\mu}, 0,0\right)
$$

Theorem1 a) The non-infected steady state $\left(T_{s s}, T^{*}(a)_{s s}, V_{s s}\right)$, when $\varphi(a)<1$, and

Is locally asymptotic stability.

$$
k<\frac{c \mu}{A \int_{0}^{\infty} p(a) \sigma(a) d a}
$$

b) When $\varphi(a)<1$, and

$$
k>\frac{c \mu}{A \int_{0}^{\infty} p(a) \sigma(a) d a}
$$

The infected steady state $\left(\bar{T}, \overline{T^{*}(a)}, \bar{V}\right)$ is locally asymptotic stability.

Proof: First, note that the general solution for $T^{*}$ can be immediately determined using characteristics as

$$
T^{*}(a, t)=\left\{\begin{array}{l}
\frac{\varphi(0)}{\varphi(a)} T^{*}\left(0, t-G(a) \exp \left(-\int_{0}^{a} \frac{\delta(s)}{\varphi(s)} d s\right), G(a)<t\right. \\
\left.\frac{\varphi\left(G^{i n v}(G(a)-t)\right.}{\varphi(a)} T^{*}\left(G^{i n v} G(a)-t\right), 0\right) \exp \left(-\int_{G(a)-t}^{a} \frac{\delta(s)}{\varphi(s)} d s\right), G(a)>t
\end{array}\right.
$$

Substituting (6) into (1), we obtain 


$$
\left\{\begin{array}{l}
\frac{d T(t)}{d t}=A-\mu T(t)-k V(t) T(t), \\
\frac{d V(t)}{d t}=\int_{0}^{t} p(a) \sigma(a) B\left(t-G(a) d a-c V(t)+F_{1}(t),\right. \\
B(t)=k V(t) T(t) .
\end{array}\right.
$$

Where

$$
\begin{aligned}
& F_{1}(t)=\int_{t}^{\infty} p(a) \frac{\phi\left(G^{i n v}(G(a)-t)\right)}{\phi(a)} \\
& \cdot T^{*}\left(G^{i n v}(G(a)-t), 0\right) \exp \left(\int_{G(a)-t}^{a} \frac{\delta(s)}{\phi(s)} d s\right) d a
\end{aligned}
$$

We can write (7) as

$$
\left\{\begin{array}{l}
\frac{d T(t)}{d t}=A-\mu T(t)-k V(t) T(t), \\
\frac{d V(t)}{d t}=K_{1} * B(t)-c V(t), \\
B(t)=k V(t) T(t) .
\end{array}\right.
$$

Where $K_{1}\left(a_{1}\right)=p(a) \sigma(a) \varphi(a), a_{1}=G(a), K_{1} * B(t)=\int_{0}^{\infty} K_{1}\left(a_{1}\right) B\left(t-a_{1}\right) d a_{1}$, And the point $\left(T_{s s}, V_{s s}, B_{s s}\right)$ of (9) is satisfied

$$
\left\{\begin{array}{l}
A_{s s}-\mu T_{s s}-k V_{s s} T_{s s}=0, \\
K_{1} * B_{s s}-c V_{s s}=0, \\
k V_{s s} T_{s s}-B_{s s}=0 .
\end{array}\right.
$$

Give us the points about which to linearism. Linear zing about any given fixed point

$$
(\bar{T}, \bar{V}, \bar{B})=\left(T-T_{s s}, V-V_{s s}, B-B_{s s}\right)
$$

Yields

$$
\left\{\begin{array}{l}
\frac{d \bar{T}}{d t}=-\mu \bar{T}-k T_{s s} \bar{V}-k V_{s s} \bar{T}, \\
\frac{d \bar{V}}{d t}=\int_{0}^{\infty} K_{1}\left(a_{1}\right) \bar{B}\left(t-a_{1}\right) d a_{1}-c \bar{V}, \\
\bar{B}=k T_{s s} \bar{V}+k V_{s s} \bar{T} .
\end{array}\right.
$$

Taking the Laplace transform of (11) we get, 


$$
\begin{aligned}
& \left\{\begin{array}{l}
-\left(\lambda+\mu+k V_{s s}\right) \hat{T}(\lambda)-k T_{s s} \hat{V}(\lambda)=T(0), \\
-(\lambda+c) \hat{V}(\lambda)+\hat{B}(\lambda) \hat{K}_{1}(\lambda)=V(0), \\
k T_{s s} \hat{V}(\lambda)+k V_{s s} \hat{T}(\lambda)-\hat{B}(\lambda)=0 .
\end{array}\right. \\
& A(\lambda)=\left(\begin{array}{ccc}
-\mu-k V_{s s}-\lambda & -k T_{s s} & 0 \\
0 & -c-\lambda & K_{1}(\lambda) \\
k V_{s s} & k T_{s s} & -1
\end{array}\right)
\end{aligned}
$$

We can see that the absence of the $\lambda$ in the $(3,3)$ element of the matrix. On taking the inverse Laplace transform, $\lambda$ are at the zeroes of $\operatorname{det}(A)$, that is, the solutions of

$$
\begin{gathered}
\left(\lambda+\mu+k V_{s s}\right)\left(\lambda+c-k T_{s s} \hat{K}_{1}(\lambda)\right) \\
+k^{2} T_{s s} V_{s s} \hat{K}_{1}(\lambda)=0
\end{gathered}
$$

The characteristic equation of Jacobian matrix at the non-infected steady state

$$
(\lambda+\mu)\left(\lambda+c-k \frac{A}{\mu} \hat{K}_{1}(\lambda)\right)=0
$$

The roots of this equation are $\lambda_{1}=-\mu$, and solutions of $\lambda+c-k \frac{A}{\mu} \hat{K}_{1}(\lambda)=0$

Hence, when $\operatorname{Re} \lambda \geq 0, \varphi(a) \leq 1$, and

$$
k<\frac{c \mu}{A \int_{0}^{\infty} p(a) \sigma(a) d a}
$$

There is a non-infected steady state, so that only when the non-infected steady state $\left(\frac{A}{\mu}, 0,0\right)$ exists, it is locally asymptotically stable.

To study the infected steady state,

$$
(\bar{T}, \bar{V}, \bar{B})=\left(\frac{c}{k N}, \frac{A}{c} N-\frac{\mu}{k}, A-\frac{\mu c}{k N}\right)
$$

There, we write

$$
N=\int_{0}^{\infty} p(a) \sigma(a) d a
$$

This yields the value equation

$$
\operatorname{det}\left(\begin{array}{ccc}
-\mu-k \bar{V}-\lambda & -k \bar{T} & 0 \\
0 & -c-\lambda & \hat{K}_{1}(\lambda) \\
-k \bar{V} & k \bar{T} & -1
\end{array}\right)=0
$$

Solving gives

The same as

$$
\lambda^{2}+\left(\frac{k A}{c} N+c-\frac{c \hat{K}_{1}(\lambda)}{N}\right) \lambda+k A N-\frac{c \mu \hat{K}_{1}(\lambda)}{N}=0
$$

$$
(\lambda+\mu)(\lambda+c)+(\lambda+c) k \bar{V}=k \bar{T}(\lambda+\mu) \hat{K}_{1}(\lambda),(\lambda+c)+\frac{(\lambda+c) k \bar{V}}{(\lambda+\mu)}=k \bar{T} \hat{K}_{1}(\lambda) .
$$

When $\operatorname{Re} \lambda \geq 0$, And $\varphi(a) \leq 1$, It means that equation has no positive real part of the 
root, so when $\varphi(a) \leq 1$, and

$$
k>\frac{c \mu}{A \int_{0}^{\infty} p(a) \sigma(a) d a}
$$

The infected steady state exists, and it is locally asymptotically stable.

In this paper, when considering the disease rate change function is not constant and $\delta(a)$ and $p(a)$ is constant, we analyze the forest diseases and insect pests model which has a wage structure of forest with disease rate change function. We obtain the conditions of the existence and the local asymptotic stability of the non-infected steady state and the infected steady state of the model. And when the infection rate of the tree infected with pests exceeds the threshold, the non-infected steady state is unstable.

\section{Acknowledgment}

Finally, thanks for the support of the National Natural Science Foundation of China (61273016).

\section{References}

[1] X. H. Xu, Z. T. Huang, L. Zen, Stability analysis of HIV - 1 model with age structured HIV / AIDS. Journal of Guangxi Teachers Education University (Natural Science Edition), 2016, 33(2):43-47.

[2]X. Z. Li, H. X. Wang, Analysis of HIV virus model with CD4 + T - cell infection in infected age structure. Journal of Applied Mathematics, 2009, 32(02):207-224

[3] Nelson Patrick W, Gilchrist Michael A, Coombs Daniel, Hyman James M, Perelson Alan S. An age-structured model of HIV infection that allows for variations in the production rate of viral particles and the death rate of productively infected cells. Mathematical Biosciences and Engineering, 2004, 1(2):267-288.

[4] Q. S. Lu, Ordinary Differential Equations and Dynamical Systems[M]. Beijing: Beijing University of Aeronautics and Astronautics Press, 2010.

[5] Y. Y. Gai, G. J. Bao, Complex Function and Integral Transformation[M]. Beijing: Science Press, 2007.

[6] D. J. Wang, Applying partial differential equations [M]. Hangzhou: Zhejiang University Press, 2007.

[7] Heathcoat H W. The Mathematics of Infectious Diseases. Siam Review, 2000, 42(4):599-653.

[8] L. Yao, H. Y. Zhu, Stability Analysis of HIV Infection Model with Age Structure. Journal of Bio-mathematics, 2012, 27(1):157-167.

[9] Y. Y. Zhang, Dynamic model of forest pests and diseases in nonlinear time delay [D].Zhejiang University of Technology, 2013. 